\title{
Nadeldekompression des Thorax
}

Andreas Höch, Niels Hammer, Philipp Brandmaier, Christoph Josten, Johannes Fakler

\section{Kasuistik}

Ein 53-jähriger polytraumatisierter Patient ist seit 13 Tagen in intensivmedizinischer Behandlung. Nach einem Verkehrsunfall erlitt der Patient neben diversen Extremitätenfrakturen und einer Wirbelsäulenverletzung auch ein Thoraxtrauma mit Fraktur der 7. und 8. Rippe rechts sowie Lungenkontusionen. Bei vorbestehender COPD verschlechterte sich die pulmonale Situation und der Allgemeinzustand des Patienten deutlich, sodass neben einer nichtinvasiven Beatmung auch die Anlage eines zentralvenösen Katheters (ZVK in die V. subclavia dextra) notwendig wurde. Eine angefertigte Thoraxübersicht nach ZVK-Anlage war unauffällig. Aufgrund einer weiteren respiratorischen Verschlechterung bestand zudem die Indikation zur Intubation, welche problemlos durchgeführt werden konnte. In den nachfolgenden 10 min kam es zu einer zunehmenden Beeinträchtigung der kardiozirkulatorischen Funktion und zum Anstieg der Beatmungsparameter. In der anschließend durchgeführten Computertomografie (CT) des
Thorax zeigt sich der in Abb. 1 dargestellte Befund. Es wurde noch im CT-Gerät eine Notfalldekompression in Monaldi-Position rechts durchgeführt. Nach dieser Notfallintervention kam zu einer deutlichen Besserung der kardiopulmonalen Situation. Nach dem Eintreffen auf der Intensivstation folgte unmittelbar die Minithorakotomie mit Einlage einer Thoraxsaugdrainage in Bülau-Position.

\section{Ziel und Zweck der Notfalltechnik}

Die Notfalldekompression des Thorax ist eine lebensrettende Sofortmaßnahme im Rahmen eines diagnostizierten oder vermuteten Spannungspneumothorax [1]. Der Spannungspneumothorax ist eine relativ selten auftretende Sonderform des Pneumothorax. Sicher ist, dass diese Verletzung unbehandelt innerhalb von Minuten zum Tod führen kann [2]. Bei penetrierenden Verletzungen oder durch Überdruckbeatmung induziert, kann durch einen Ventilmechanismus der verletzten Lunge

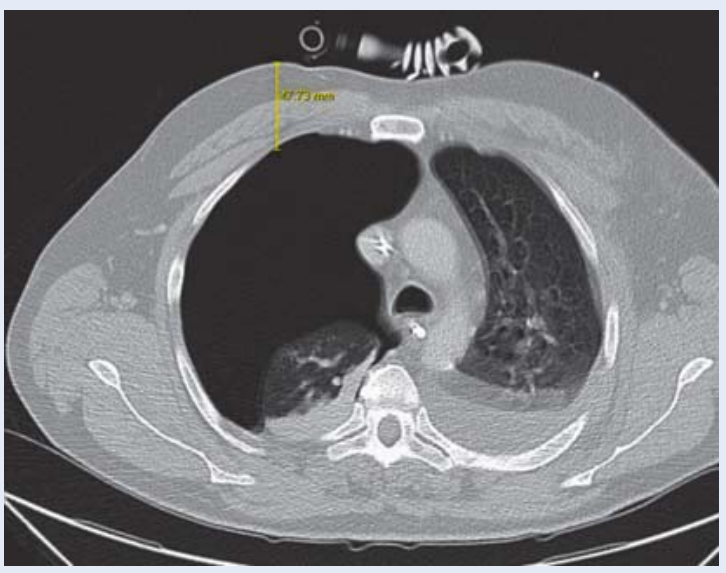

Abb. 1 53-jähriger Patient. Dargestellt ist der CT-Schnitt auf Höhe des 2. ICR bei bestehendem Spannungspneumothorax rechts. Messung der Thoraxwanddicke von $4,8 \mathrm{~cm}$ im Bereich der Punktionsstelle der Nadeldekompression. bzw. der verletzten Thoraxwand Luft während der Inspiration in den Pleuraspalt eindringen. Kann diese Luft nicht mehr entweichen, steigt der intrathorakale Druck kontinuierlich an und beeinträchtigt die pulmonale sowie im weiteren Verlauf auch die kardiozirkulatorische Funktion [2-4]. In der präklinischen Notfalltherapie muss die Diagnose des Spannungspneumothorax rein klinisch erkannt werden, da keine Bildgebung zur Verfügung steht.

Die exakte Inzidenz des Spannungspneumothorax ist unklar. Man geht davon aus, dass bei etwa $5 \%$ der schwerverletzten Patienten mit Thoraxtrauma ein Spannungspneumothorax auftritt, insbesondere wenn eine mechanische Beatmung erforderlich ist [6]. Die Letalität des Spannungspneumothorax im präklinischen Bereich liegt allerdings unter $0,3 \%$, da die meisten Patienten mit stumpfem Thoraxtrauma wach sind [7]. Andererseits zeigt eine Autopsiestudie, dass ein unbehandelter Spannungspneumothorax als wesentliche Ursache für einen potenziell vermeidbaren Todesfall beim Traumapatienten angesehen werden kann [8].

Ist die (Verdachts-)Diagnose eines Spannungspneumothorax gestellt, muss die Dekompression des Pleuraraumes unmittelbar durchgeführt werden. Zur Verfügung steht zum einen die Technik der chirurgischen Minithorakotomie, mit oder ohne Einlage einer Drainage, zum anderen die Nadeldekompression. Die Vor- und Nachteile dieser Verfahren werden in der Literatur kontrovers diskutiert. Zudem existieren keine Studien mit größeren Fallzahlen, wodurch ein direkter Vergleich schwierig ist. In den S3-Leitlinie „Polytrauma/Schwerverletz- 
Symptome und klinische Befunde bei Spannungspneumothorax

$[2,3,5]$

- thorakaler Schmerz

- Dyspnoe

- abgeschwächtes oder aufgehobenes Atemgeräusch/Beatmungsgeräusch (nach Tubuslagekontrolle)

- hypersonorer Klopfschall

- obere Einflussstauung

- Kreislaufdepression (Tachykardie, Hypotension)

- hohe Beatmungsdrücke bei maschineller Beatmung

tenbehandlung" und auch im Kurshandbuch „Advanced Trauma and Life Support“ $\left(\right.$ ATLS $\left.^{\circledR}\right)$ wird empfohlen, dass nach einer Nadeldekompression bereits prähospital zusätzlich die Anlage einer Thoraxsaugdrainage zu erfolgen hat $[3,9]$.

Der Spannungspneumothorax ist die einzige vitale Indikation für die Nadeldekompression des Thorax. Geht man im Notfall von einem Spannungspneumothorax aus, so bestehen per definitionem keine Kontraindikationen zur Punktion. Die Vorteile liegen in der schnellen und relativ einfachen Durchführung, was v.a. im amerikanischen Raum zur Umsetzung bei Militäreinsätzen auch durch nichtärztliches Personal - führt. Untersuchungen konnten eine Verkürzung der präklinischen Behandlungszeit um 5 Minuten im Vergleich zur Minithorakotomie nachweisen $[10,11]$.

Aktuell gehen die Empfehlungen zur technischen Durchführung der Nadeldekompression und zur Wahl des Punktionsortes auseinander. Zur Punktion wird der 2. oder 3. Interkostalraum (ICR) in der mittleren
Klavikularlinie empfohlen. Aber auch der 4 . oder 5. ICR wird in der vorderen Axillarlinie von einigen Autoren bevorzugt, da hier eine geringere Thoraxwanddicke gezeigt werden konnte [12]. Im deutschsprachigen Raum wird der 2. ICR in der mittleren Klavikularlinie bevorzugt. Ebenso umstritten ist die zu verwendende Nadellänge. In den Leitlinien des U.S.-Militärs wird die Nutzung von 8,2 bzw. $8,9 \mathrm{~cm}$ langen Nadeln gefordert. Dahingegen wird in der deutschen S3-Leitlinie „Polytrauma/Schwerverletztenbehandlung“ bei fehlender Risikoabschätzung nicht empfohlen, Nadeln mit mehr als 4,5 cm Länge zu nutzen $[3,13]$. Man geht davon aus, dass die Zahl iatrogener Verletzungen bei längeren Nadeln deutlich steigen kann. Radiologische Auswertungen sehen v.a. die Herzkammern, aber auch die großen thorakalen Gefäße in Gefahr. Im Widerspruch zur empfohlenen Nadellänge von $4,5 \mathrm{~cm}$ verweisen mehrere Untersuchungen aber auch auf eine hohe Versagensrate bei der Verwendung von $4,5 \mathrm{~cm}$ langen Nadeln, da der Pleuraspalt hierdurch nicht erreicht wird $[4,11,13]$.

\section{Fazit}

Ohne hohes Evidenzlevel kann man folgende aktuelle Punkte zur Durchführung der Nadeldekompression des Thorax für den deutschsprachigen Raum zusammenfassen $[3,4,9]$ :

- Indikation zur Nadelpunktion besteht bei vermutetem oder diagnostiziertem Spannungspneumothorax

- im Notfall bestehen keine Kontraindikationen

- eine zusätzliche Minithorakotomie sollte im Anschluss immer erfolgen

- Punktionsort der Wahl ist der 2. ICR in der mittleren Klavikularlinie

- die Nadellänge sollte $4,5 \mathrm{~cm}$ bei 14 Gauge Durchmesser betragen

\section{Komplikationen im Rahmen der Nadeldekompression}

- Verletzung intrathorakaler Strukturen (z. B. Lunge, Gefäße, Trachea)

- intrathorakale Blutung (z. B. durch Verletzungen der Lunge, Interkostalgefäße, des Herzens)

- Ineffektivität der Maßnahme bei Nichterreichen des Pleuraraumes (Nichtentlastung des Spannungspneumothorax)

- Verlegung des Lumens der Punktionsnadel/Venenverweilkanüle durch Dislokation, anliegendes Lungengewebe oder Blut und

- Risiko einer erneuten Entstehung eines Spannungspneumothorax nach initialer Entlastung 


\section{Nadeldekompression des Thorax}

\section{Schritt für Schritt}
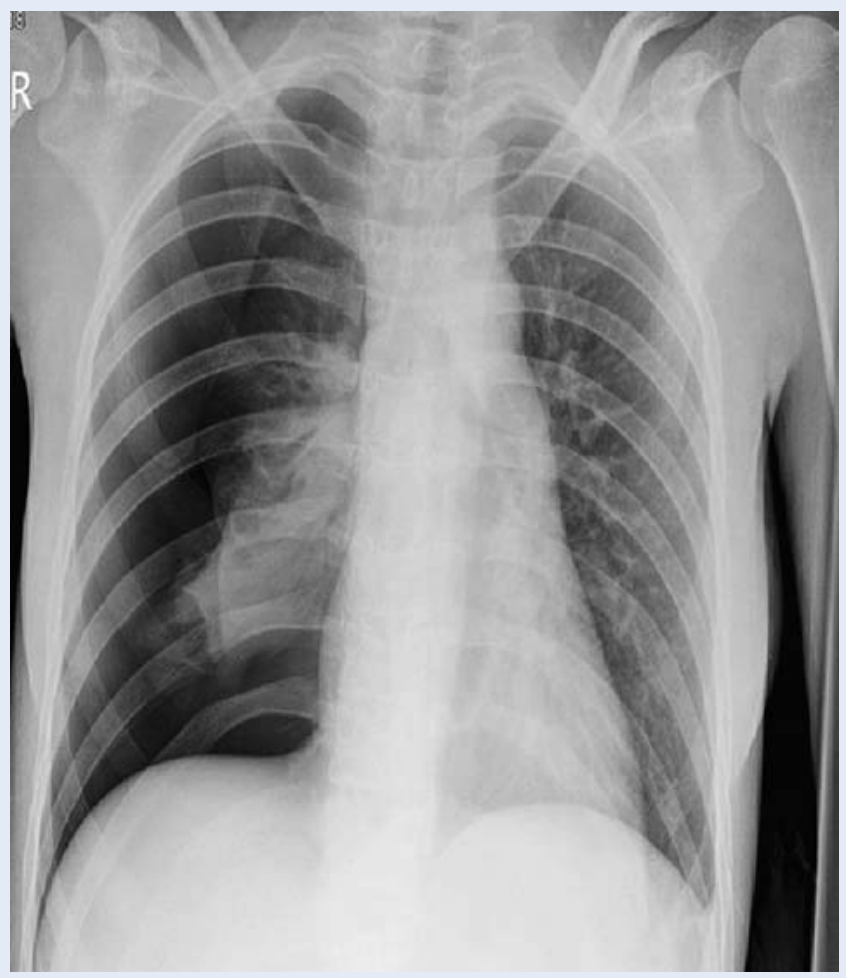

Abb. 2 42-jähriger Patient mit Spannungspneumothorax. Klinisch beginnende Kreislaufdepression, aufgehobenes Atemgeräusch rechts. Radiologisches Vollbild eines Spannungspneumothorax (vollständig kollabierte Lunge rechts, Zwerchfelltiefstand rechts, Mediastinalverschiebung nach links).

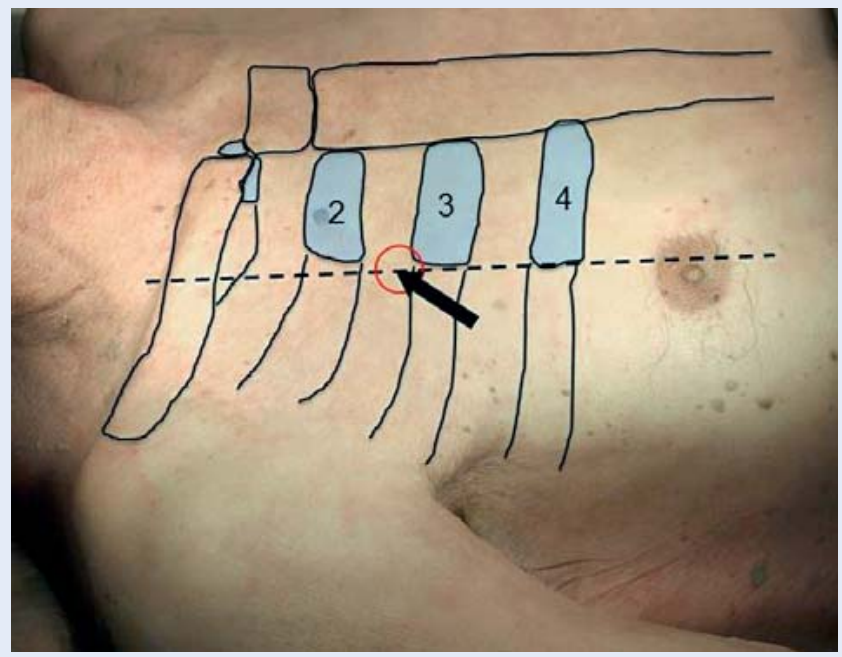

Abb. 4 Die Notfalldekompression wird im 2. Interkostalraum auf der mittleren Klavikularlinie in der Monaldi-Position empfohlen. Hierfür ist die meist gut tastbare Klavikula und die 2. und 3. Rippe aufzusuchen. Die 1. Rippe ist in der Regel nicht von ventral zu tasten. Im Bild rot markiert ist der gewünschte Punktionsort.

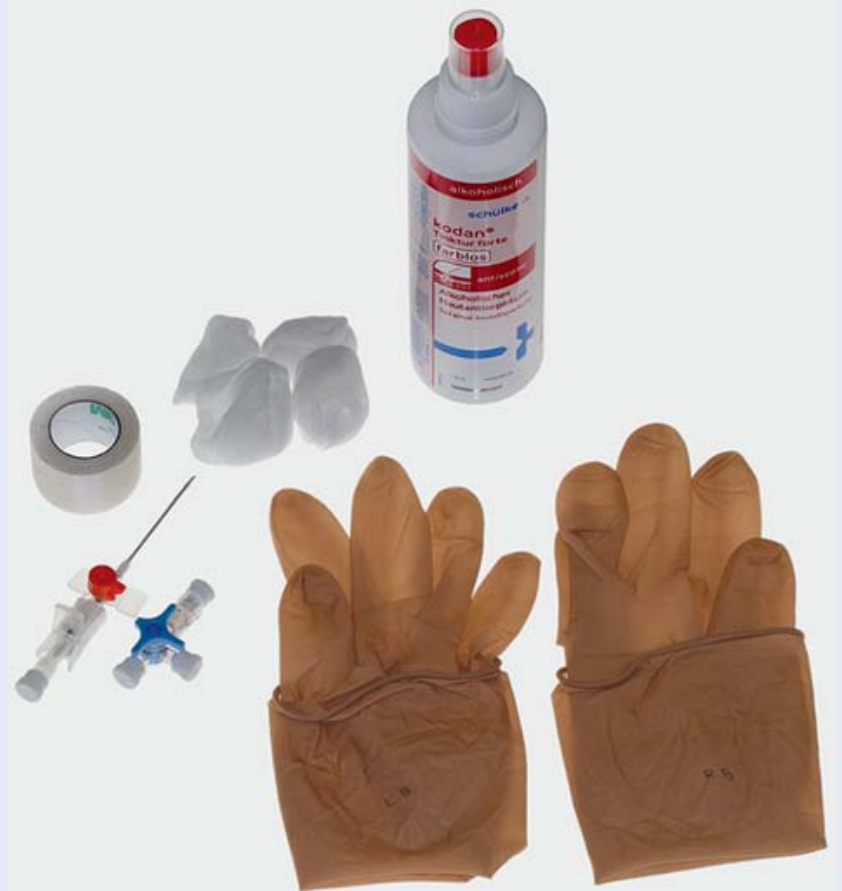

Abb. 3 Notwendiges Zubehör für eine Nadeldekompression bei Spannungspneumothorax: Desinfektionsmittel, sterile Handschuhe, 14-Gauge-Venenverweilkanüle, Drei-Wege-Hahn, Tupfer und Pflaster zur sicheren Fixierung.

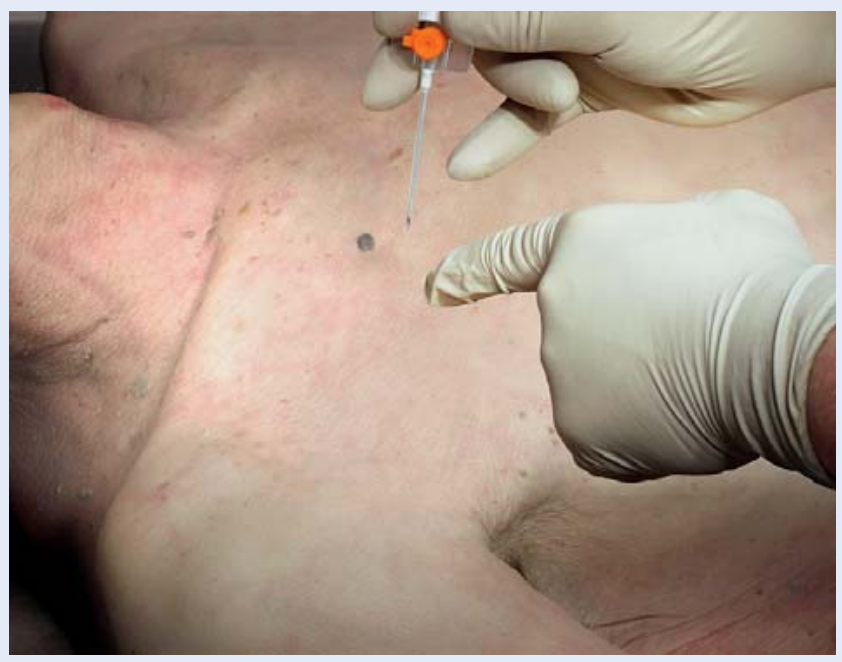

Abb. 5 Nach einer gründlichen Sprühdesinfektion sollte mit der freien Hand die 3. Rippe palpiert und die Kanüle mit der anderen Hand streng am Oberrand der Rippe senkrecht eingestochen werden. Die Kanüle wird in ihrer gesamten Länge eingeführt und der Stahlmandrin entfernt. Bei Erreichen des Pleuraspalts und vorliegendem Spannungspneumothorax kommt es zu einem hörbaren „Zischen“ in Folge des Luftaustritts. 


\section{Schritt für Schritt}

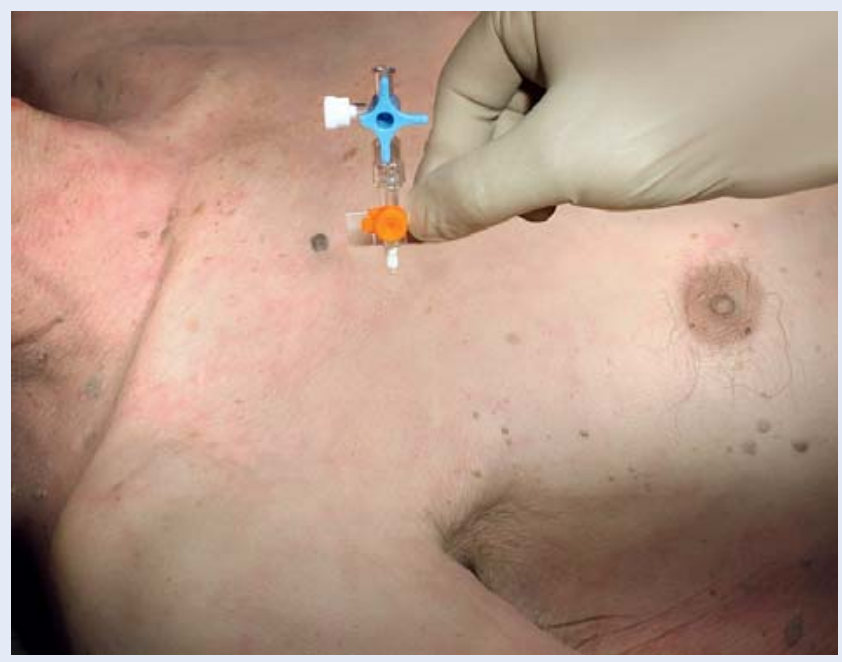

Abb. 6 Sinnvoll erscheint auch die Nutzung eines Drei-Wege-Hahns, um die Nadel einfach verschließen und wieder öffnen zu können.

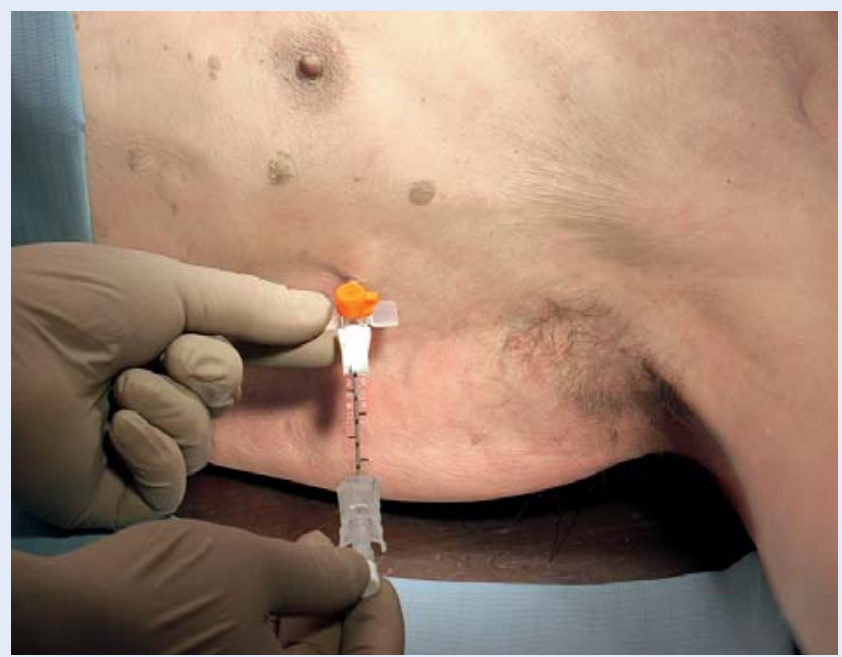

Abb. 8 Analog zur Nadeldekompression in der Monaldi-Position kann auch die Position nach Bülau im 4. oder 5. ICR in der vorderen Axillarlinie verwendet werden. Sinnvoller ist es jedoch, diesen Bereich der Minithorakotomie und Thoraxdrainageneinlage vorzubehalten. Im Falle der Nadeldekompression ist auch hier streng am Oberrand der jeweiligen Rippe zu punktieren.

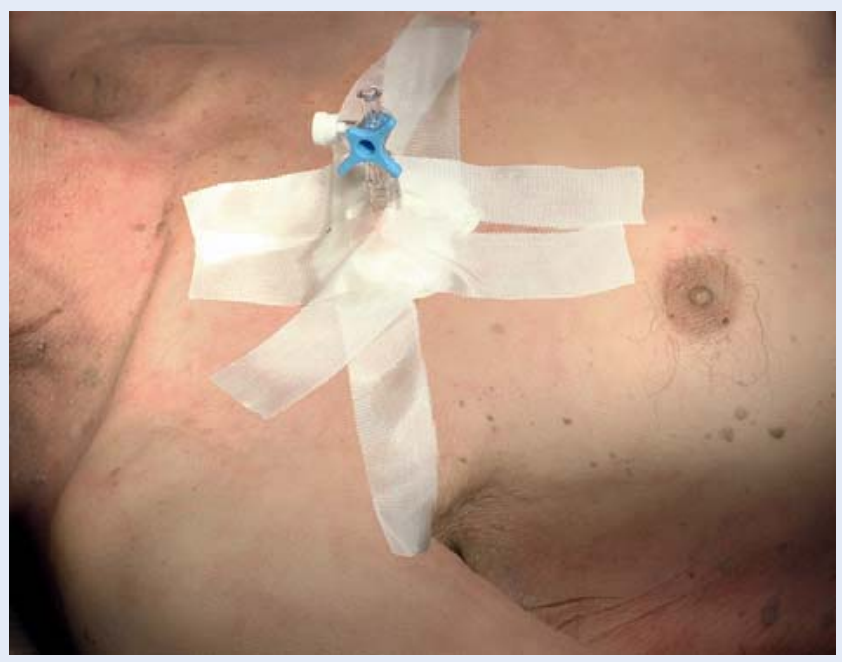

Abb. 7 Sehr wichtig und schnell vernachlässigt ist die sorgfältige Fixierung der liegenden Nadel. Nach 2 parallel angeordneten Pflasterstreifen über die Auflageflächen der Venenverweilkanüle sollten zudem noch oberhalb und unterhalb der Kanüle angedeutet u-förmig 2 weitere Pflasterstreifen angebracht werden. Gerade im Notfalleinsatz kann es durch Lagerung, Transport oder andere Maßnahmen schnell zu einer Dislokation und folgenden Insuffizienz der Nadel kommen.

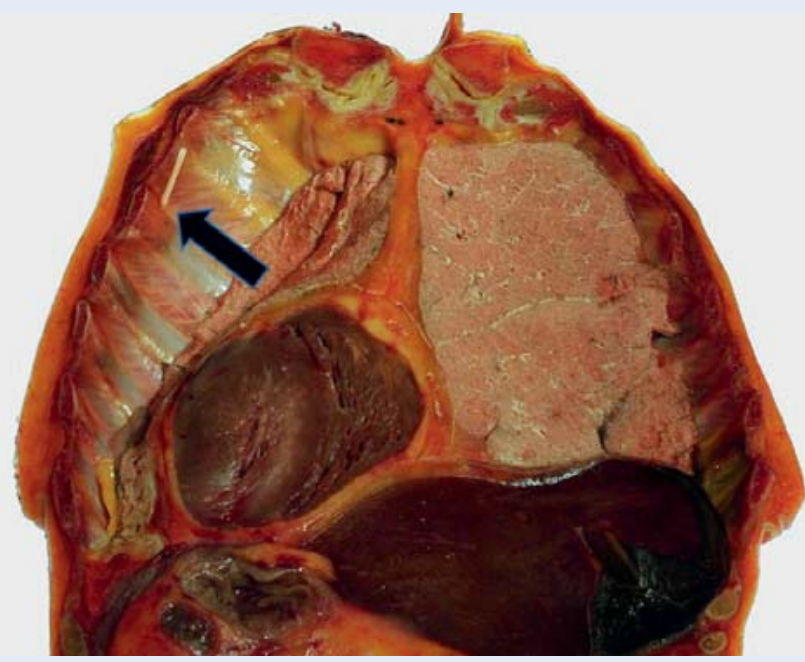

Abb.9 Intrathorakale Lage der Venenverweilkanüle. Sicht von innen auf die Thoraxvorderwand mit simuliertem Pneumothorax links. 
Interessenkonflikt: Die Autoren geben an, dass kein Interessenkonflikt besteht.

\section{Literatur}

1 Grabo D, Inaba K, Hammer P et al. Optimal training for emergency needle thoracostomy placement by prehospital personnel: didactic teaching versus a cadaver-based training program. J Trauma Acute Care Surg 2014; 77: 109-113

2 Leigh-Smith S, Harris T. Tension pneumothoraxtime for a re-think? Emerg Med J 2005; 22: 8-16

3 Deutsche Gesellschaft für Unfallchirurgie. S3-Leitlinie Polytrauma/Schwerverletzten-Behandlung (2011). Im Internet: http://www.awmf.org/leitlinien/detail/II/012-019.html; Stand: 23.02.2015

4 Hockertz DK, Braun KF, Trentzsch H. Nadeldekompression des Spannungspneumothorax. Notfall Rettungsmed 2015; 1: 49-52

5 Barton ED. Tension pneumothorax. Curr Opin Pulm Med 1999; 5: 269-274

6 Coats TJ, Wilson AW, Xeropotamous N. Pre-hospital management of patients with severe thoracic injury. Injury 1995; 26: 581-585

7 Leigh-Smith S. Tension pneumothorax prevalence grossly exaggerated. Emerg Med J 2007; 24: 865

8 Kleber C, Giesecke MT, Tsokos M et al. Trauma-related preventable deaths in Berlin 2010: need to change prehospital management strategies and trauma management education. World J Surg 2013; 37: 1154-1161

9 American College of Surgeons, eds. ATLS, advanced trauma life support for doctors. 8th ed. Chicago, IL: American College of Surgeons; 2008

10 Cantwell K, Burgess S, Patrick I et al. Improvement in the prehospital recognition of tension pneumothorax: the effect of a change to paramedic guidelines and education. Injury 2014; 45: 71-76

11 Martin M, Satterly S, Inaba K et al. Does needle thoracostomy provide adequate and effective decompression of tension pneumothorax? J Trauma Acute Care Surg 2012; 73: 1412-1417

12 Inaba K, Branco BC, Eckstein M et al. Optimal positioning for emergent needle thoracostomy: a cadaver-based study. J Trauma 2011; 71: 1099-1103

13 Chang SJ, Ross SW, Kiefer DJ et al. Evaluation of 8.0$\mathrm{cm}$ needle at the fourth anterior axillary line for needle chest decompression of tension pneumothorax. J Trauma Acute Care Surg 2014; 76: 10291034

\section{Über die Autoren}

Andreas Höch

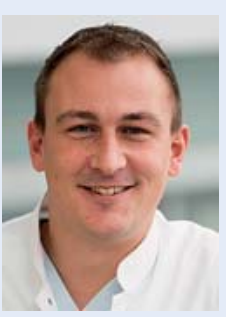

\section{Niels Hammer}

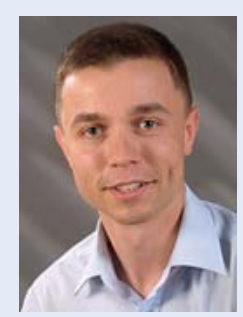

Wissenschaftlicher Mitarbeiter am Institut für Anatomie, Universität Leipzig.

\section{Philipp Brandmaier}

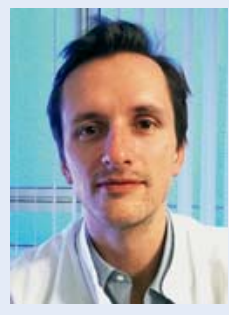

Assistenzarzt der Klinik und Poliklinik für Diagnostische und Interventionelle Radiologie am Universitätsklinikum Leipzig.

\section{Christoph Josten}

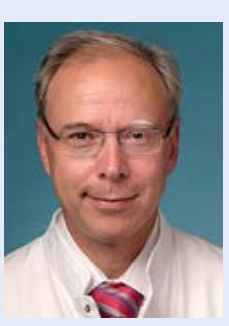

Geschäftsführender Klinikdirektor der Klinik für Orthopädie, Unfallchirurgie und Plastische Chirurgie am Universitätsklinikum Leipzig und leitender Notarzt.

\section{Johannes Fakler}

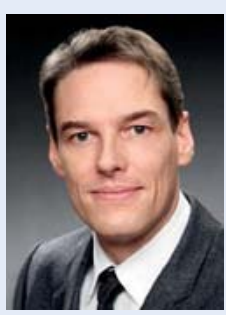

Seit 2010 Oberarzt an der Klinik für Orthopädie, Unfallchirurgie und Plastische Chirurgie am Universitätsklinikum Leipzig und Notarzt.

\section{Korrespondenzadresse}

Dr. med. Johannes Fakler

Klinik für Orthopädie, Unfallchirurgie

und Plastische Chirurgie

Universitätsklinikum Leipzig, AöR

Liebigstraße 20

04103 Leipzig

E-Mail: johannes.fakler@medizin.uni-leipzig.de

Koordination der Rubrik „Schritt für Schritt“:

PD Dr. med. Michael Bernhard, Leipzig 